\title{
Disk-cyclic and Codisk-cyclic tuples of the adjoint weighted composition operators on Hilbert spaces*
}

\author{
Yu-Xia Liang Ze-Hua Zhou ${ }^{\dagger}$
}

\begin{abstract}
Some sufficient conditions under which the tuple of the adjoint of weighted composition operators $\left(C_{\omega_{1}, \varphi_{1}}^{*}, C_{\omega_{2}, \varphi_{2}}^{*}\right)$ on the Hilbert space $\mathcal{H}$ of analytic functions is disk-cyclic (or codisk-cyclic) were investigated.
\end{abstract}

\section{Introduction}

Let $\mathcal{H}$ be an infinite dimensional separable Hilbert space of analytic functions defined in $\mathbb{D}=\{z \in \mathbb{C},|z|<1\}$ such that, for each $\lambda \in \mathbb{D}$, the linear functional of point evaluation $e_{\lambda}(f)=f(\lambda)$ is bounded. The Riesz representation theorem states that $e_{\lambda}(f)=\left\langle f, k_{\lambda}\right\rangle$ for some $k_{\lambda} \in \mathcal{H}$, the reproducing kernel of $\mathcal{H}$. The collection of all holomorphic functions (or self-maps) in $\mathbb{D}$ is denoted as $H(\mathbb{D})$ (or $S(\mathbb{D})$ ). Recently, hypercyclic and supercyclic operators have received considerable attention, especially since they arise in familiar classes of operators, such as weighted shifts $[5,6,12,13,14,20]$, composition operators [15], weighted composition operators [4, 11, 16, 19, 21, 22, 23]. For motivation, examples and

\footnotetext{
* The work was supported in part by the National Natural Science Foundation of China (Grant Nos. 11371276; 11301373; 11401426) the Doctoral Fund of Tianjin Normal University (Grant Nos. 52XB1514).

†Corresponding author.

Received by the editors in May 2015 - In revised form in November 2015.

Communicated by F. Bastin.

2010 Mathematics Subject Classification : Primary: 47A16; Secondary: 47B33, 47B38, 46E15.

Key words and phrases : Disk-cyclic, codisk-cyclic, adjoint, weighted composition operator, Hilbert space.
} 
background about linear dynamics, we refer the readers to the excellent books [2] by Bayart and Matheron, [7] by Grosse-Erdmann and Peris Manguillot.

For a backward shift $B$ on $\ell^{p}(\mathbb{N})$, Rolewicz [18] showed that $\lambda B$ is hypercyclic if and only if $|\lambda|>1$. Hence $\lambda B$ is not hypercyclic whenever $|\lambda| \leq 1$, one may wonder if there is an operator $\widetilde{T}$ satisfying its disk-orbit is dense in a Hilbert space. Along with this question, a new dynamical property-diskcyclicity emerged in $[1,8,9,10,17]$. To be specific, an operator $\widetilde{T} \in \mathcal{B}(\mathcal{H})$ is disk-cyclic (or codisk-cyclic), if there exists a vector $x \in \mathcal{H}$ such that $\operatorname{DOrb}(\widetilde{T}, x)=$ $\left\{\alpha \widetilde{T}^{n} x: \alpha \in \mathbb{C}, 0<|\alpha| \leq 1, n \geq 0\right\}$ (or $\left\{\beta \widetilde{T}^{n} x: \beta \in \mathbb{C},|\beta| \geq 1, n \geq 0\right\}$ ) is norm-dense in $\mathcal{H}$ and $x$ is called a disk-cyclic (or codisk-cyclic) vector for $\widetilde{T}$, where $\widetilde{T}^{n}$ is obtained by composing $\widetilde{T}$ with itself $n$ times.

An $n$-tuple of operators acting on $\mathcal{H}$ is a finite sequence of length $n$ of commuting continuous linear operators $T_{1}, T_{2}, \cdots, T_{n}$ on $\mathcal{H}$, and is written as $T=$ $\left(T_{1}, T_{2}, \cdots, T_{n}\right)$. For $T=\left(T_{1}, T_{2}, \cdots, T_{n}\right)$, we denote $\mathcal{F}=\mathcal{F}_{T}=\left\{T_{1}^{k_{1}} T_{2}^{k_{2}} \cdots T_{n}^{k_{n}}\right.$ : $\left.k_{i} \in \mathbb{N}, i=1,2, \cdots, n\right\}$, which is the semigroup generated by $T$. Given $x \in \mathcal{H}$, the orbit of $x$ under the tuple $T$ is $\operatorname{Or} b(T, x)=\{S x: S \in \mathcal{F}\}$. Naturally, $T=$ $\left(T_{1}, T_{2}, \cdots, T_{n}\right)$ is disk-cyclic (or codisk-cyclic) if there is a vector $x \in \mathcal{H}$ such that $\mathbb{D O r b}(T, x)=\{\alpha S x: \alpha \in \mathbb{C}, 0<|\alpha| \leq 1, S \in \mathcal{F}\}\left(\right.$ or $\mathbb{D}^{c} \operatorname{Orb}(T, x)=\{\beta S x: \beta \in$ $\mathbb{C},|\beta| \geq 1, S \in \mathcal{F}\}$ ) is dense in $\mathcal{H}$ and $x$ is called a disk-cyclic (or codisk-cyclic) vector for the tuple $T$.

A complex-valued function $\omega$ in $\mathbb{D}$ such that $\omega f \in \mathcal{H}$ for every $f \in \mathcal{H}$ is called a multiplier of $\mathcal{H}$ and the collection of all multipliers is denoted by $\mathcal{M}(\mathcal{H})$. In [11, p552] Kamali etc. proved $\mathcal{M}(\mathcal{H}) \subseteq H^{\infty}$. A multiplication operator $M_{\omega}$ on $\mathcal{H}$ is $M_{\omega} f=\omega f, f \in \mathcal{H}$. For $\omega \in M(\mathcal{H})$ and $\varphi \in S(\mathbb{D})$ such that $f \circ \varphi \in \mathcal{H}$ for every $f \in \mathcal{H}$, then the weighted composition operator $C_{\omega, \varphi}: \mathcal{H} \rightarrow \mathcal{H}, C_{\omega, \varphi}(f)(z)=M_{\omega} C_{\varphi}(f)(z)=\omega(z) f(\varphi(z))$ is bounded. Due to the fact $C_{\omega, \varphi}^{*}\left(k_{\lambda}\right)=\overline{w(\lambda)} k_{\varphi(\lambda)}$ for every $\lambda \in \mathbb{D}$, it yields that $C_{\omega, \varphi}^{* n}\left(k_{\lambda}\right)=$ $\left(\prod_{j=0}^{n-1} \overline{\omega\left(\varphi_{j}(\lambda)\right)}\right) k_{\varphi_{n}(\lambda)}$. Given $\omega_{1}, \omega_{2} \in \mathcal{M}(\mathcal{H})$ and $\varphi_{1}, \varphi_{2} \in S(\mathbb{D})$, we obtain $C_{\omega_{1}, \varphi_{1}}$ and $C_{\omega_{2}, \varphi_{2}}$. In the current paper, we assume that the constants and the identity function $f(z)=z$ are in $\mathcal{H}$, and denote $T=\left(T_{1}, T_{2}\right)=\left(C_{\omega_{1}, \varphi_{1}}^{*}, C_{\omega_{2}, \varphi_{2}}^{*}\right)$. Firstly, fix $A, B, C, D$ the four subsets of $\mathbb{D}$ as below,

$$
\begin{gathered}
A=\left\{z \in \mathbb{D}: \text { the sequence }\left\{\Pi_{j=0}^{n-1}\left(\omega_{1} \circ\left(\varphi_{1}\right)_{j}(z) \cdot \omega_{2} \circ\left(\varphi_{2}\right)_{j}(z)\right)\right\}_{n} \text { is bounded }\right\}, \\
B=\left\{z \in \mathbb{D}: \lim _{n \rightarrow \infty} \Pi_{j=1}^{n}\left(\omega_{1} \circ\left(\varphi_{1}\right)_{-j}(z) \cdot \omega_{2} \circ\left(\varphi_{2}\right)_{-j}(z)\right)^{-1}=0\right\}, \\
C=\left\{z \in \mathbb{D}: \lim _{n \rightarrow \infty} \Pi_{j=0}^{n-1}\left(\omega_{1} \circ\left(\varphi_{1}\right)_{j}(z) \cdot \omega_{2} \circ\left(\varphi_{2}\right)_{j}(z)\right)=0\right\}, \\
D=\left\{z \in \mathbb{D}: \text { the sequence }\left\{\Pi_{j=1}^{n}\left(\omega_{1} \circ\left(\varphi_{1}\right)_{-j}(z) \cdot \omega_{2} \circ\left(\varphi_{2}\right)_{-j}(z)\right)^{-1}\right\}_{n}\right. \\
\text { is bounded }\} .
\end{gathered}
$$

In [16, Theorem 3.1], under the assumptions in (2.2)(in section 2) and $M=\sup _{z \in \mathbb{D}} \sup _{n \in \mathbb{Z}}\left\|k_{\left(\varphi_{1}\right)_{n} \circ\left(\varphi_{2}\right)_{n}(z)}\right\|<\infty$. Either the sets $A$ and $B$ or the sets $C$ and 
$D$ have limit points in $\mathbb{D}$, then the tuple $T=\left(C_{\omega_{1}, \varphi_{1}}^{*}, C_{\omega_{2}, \varphi_{2}}^{*}\right)$ is supercyclic on $\mathcal{H}$. Inspired by the above work and the ideas in [16], we will find some sufficient conditions to guarantee the disk-cyclicity and codisk-cyclicity of the tuple $T=\left(C_{\omega_{1}, \varphi_{1}}^{*}, C_{\omega_{2}, \varphi_{2}}^{*}\right)$ on $\mathcal{H}$, which are new and interesting question for the investigation of weighted composition operators. Indeed the similar conclusions could be obtained for the $n$-tuple $T=\left(C_{\omega_{1}, \varphi_{1}}^{*}, C_{\omega_{2}, \varphi_{2}}^{*}, \cdots, C_{\omega_{n}, \varphi_{n}}^{*}\right)$, we leave the proof for the readers. The organization of the paper is as follows. In Section 2, we prepared some preliminary results, and then we showed several sufficient conditions ensuring the disk-cyclicity and codisk-cyclicity of the tuple $\left(C_{\omega_{1}, \varphi_{1}}^{*}, C_{\omega_{2}, \varphi_{2}}^{*}\right)$ in Section 3 and Section 4 , respectively.

\section{Preliminary results}

In this section, we cited and proved some lemmas which were needed in the proofs of our main results. Firstly, we cited a necessary and sufficient condition ensuring $C_{\omega_{1}, \varphi_{1}}$ and $C_{\omega_{2}, \varphi_{2}}$ to commute.

Lemma 2.1. [19, Lemma 1] If $\omega_{1}(z)$ and $\omega_{2}(z)$ are nonzero for all $z \in \mathbb{D}$, then $C_{\omega_{1}, \varphi_{1}}$ and $C_{\omega_{2}, \varphi_{2}}$ can commute if and only if

$$
\varphi_{1} \circ \varphi_{2}=\varphi_{2} \circ \varphi_{1} \text { and } \omega_{1} \cdot\left(\omega_{2} \circ \varphi_{1}\right)=\omega_{2} \cdot\left(\omega_{1} \circ \varphi_{2}\right) \text {. }
$$

Remark 2.2. Furthermore, we will assume that $\omega_{1}(z)$ and $\omega_{2}(z)$ are nonzero for all $z \in \mathbb{D}$ and $\varphi_{1}, \varphi_{2}$ satisfy

$$
\varphi_{1} \circ \varphi_{2}=\varphi_{2} \circ \varphi_{1}, \omega_{1}=\omega_{1} \circ \varphi_{2} \text { and } \omega_{2}=\omega_{2} \circ \varphi_{1} \text {. }
$$

It's easy to verify that $C_{\omega_{1}, \varphi_{1}}$ and $C_{\omega_{2}, \varphi_{2}}$ can commute under (2.2) and [19, p456] shows there are many maps satisfying (2.2).

Let $T_{i}=C_{\omega_{i}, \varphi_{i}}^{*}$ for $i=1,2$. A straightforward calculation gives that

$$
T_{i}^{n} k_{z}=\left(\Pi_{j=0}^{n-1} \overline{\left(\omega_{i} \circ\left(\varphi_{i}\right)_{j}\right)(z)}\right) k_{\left(\varphi_{i}\right)_{n}(z), \quad i=1,2, n \geq 1 .}
$$

Employing (2.2), it turns out that

$$
\begin{aligned}
& T_{2}^{n} T_{1}^{n} k_{z} \\
& =\left(\Pi_{k=0}^{n-1} \overline{\left(\omega_{2} \circ\left(\varphi_{2}\right)_{k}\right)(z)}\right)\left(\Pi_{j=0}^{n-1} \overline{\left(\omega_{1} \circ\left(\varphi_{1}\right)_{j} \circ\left(\varphi_{2}\right)_{n}\right)(z)}\right) k_{\left(\varphi_{1}\right)_{n} \circ\left(\varphi_{2}\right)_{n}(z)} \\
& =\left(\Pi_{k=0}^{n-1} \overline{\left(\omega_{2} \circ\left(\varphi_{2}\right)_{k}\right)(z)}\right)\left(\Pi_{j=0}^{n-1} \overline{\left(\omega_{1} \circ\left(\varphi_{2}\right)_{n} \circ\left(\varphi_{1}\right)_{j}\right)(z)}\right) k_{\left(\varphi_{1}\right)_{n} \circ\left(\varphi_{2}\right)_{n}(z)} \\
& =\left(\Pi_{k=0}^{n-1} \overline{\left(\omega_{2} \circ\left(\varphi_{2}\right)_{k}\right)(z)}\right)\left(\Pi_{j=0}^{n-1} \overline{\left(\omega_{1} \circ\left(\varphi_{1}\right)_{j}\right)(z)}\right) k_{\left(\varphi_{1}\right)_{n} \circ\left(\varphi_{2}\right)_{n}(z)} \\
& =\left[\Pi_{j=0}^{n-1}\left(\overline{\left(\omega_{2} \circ\left(\varphi_{2}\right)_{j}\right)(z)} \cdot \overline{\left(\omega_{1} \circ\left(\varphi_{1}\right)_{j}\right)(z)}\right)\right] k_{\left(\varphi_{1}\right)_{n} \circ\left(\varphi_{2}\right)_{n}(z)} \\
& =\left[\Pi_{j=0}^{n-1}\left(\overline{\left(\omega_{1} \circ\left(\varphi_{1}\right)_{j}\right)(z)} \cdot \overline{\left(\omega_{2} \circ\left(\varphi_{2}\right)_{j}\right)(z)}\right)\right] k_{\left(\varphi_{1}\right)_{n} \circ\left(\varphi_{2}\right)_{n}(z)} .
\end{aligned}
$$

As regards to the disk-cyclicity and codisk-cycliclity, the Disk-Cyclicity Criterion ([10, Proposition 2.5]) and the Codisk-Cyclicity Criterion([8, Proposition 5.2.9]) 
are dispensable tools in finding disk-cyclic and codisk-cyclic operators, which relate with the Supercyclicity Criterion [3]. In order to derive the corresponding criteria for tuples, we firstly presented the equivalent characterization for the disk-cyclic or codisk-cyclic tuple $T=\left(T_{1}, T_{2}\right)$ on $\mathcal{H}$ paralleling to [8, Theorem 4.24 and Theorem 5.2.4], respectively.

Theorem 2.3. Let $T=\left(T_{1}, T_{2}\right)$ be a 2-tuple of continuous linear operators on $\mathcal{H}$.

(1) For the disk-cyclicity, the following statements are equivalent:

(i) The tuple $T=\left(T_{1}, T_{2}\right)$ is disk-cyclic.

(ii) For each $x, y \in \mathcal{H}$ and each neighborhood $W$ for zero in $\mathcal{H}$, there are $z \in \mathcal{H}$, $k_{1}, k_{2} \in \mathbb{N}, \alpha \in \mathbb{C}$ with $0<|\alpha| \leq 1$ such that $x-z \in W$ and $T_{1}^{k_{1}} T_{2}^{k_{2}} \alpha z-y \in W$.

(2) For the codisk-cyclicity, the following statements are equivalent:

(i') The tuple $T=\left(T_{1}, T_{2}\right)$ is codisk-cyclic.

(ii') For each $x, y \in \mathcal{H}$ and each neighborhood $W$ for zero in $\mathcal{H}$, there are $z \in \mathcal{H}$, $k_{1}, k_{2} \in \mathbb{N}, \beta \in \mathbb{C}$ with $|\beta| \geq 1$ such that $x-z \in W$ and $T_{1}^{k_{1}} T_{2}^{k_{2}} \beta z-y \in W$.

In the following, we verified the Disk-Cyclicity Criterion and the Codiskcyclicity Criterion for the tuple $T=\left(T_{1}, T_{2}\right)$ by Theorem 2.3(1) and (2), respectively.

Proposition 2.4. (Disk-Cyclicity Criterion for tuples) Let $\mathcal{H}$ be a separable infinite dimensional Hilbert space and $T=\left(T_{1}, T_{2}\right)$ be a pair of commuting continuous linear mappings on $\mathcal{H}$. If there exist two dense subsets $\mathcal{X}, \mathcal{Y}$ in $\mathcal{H}$, a pair of strictly increasing positive integer sequences $\left(m_{k}\right)_{k \in \mathbb{N}}$ and $\left(n_{k}\right)_{k \in \mathbb{N}}$ and a sequence of mappings $S_{k}$ (not necessary bounded) such that $S_{k}(\mathcal{Y}) \subset \mathcal{Y}$ and

(1) $T_{1}^{m_{k}} T_{2}^{n_{k}} S_{k} y \rightarrow y, k \rightarrow \infty$, and $\lim _{k \rightarrow \infty}\left\|S_{k} y\right\|=0$ for all $y \in \mathcal{Y}$;

(2) $\lim _{k \rightarrow \infty}\left\|T_{1}^{m_{k}} T_{2}^{n_{k}} x\right\|\left\|S_{k} y\right\|=0$ for all $x \in \mathcal{X}, y \in \mathcal{Y}$.

Then we say that $T=\left(T_{1}, T_{2}\right)$ satisfies the Disk-Cyclicity Criterion. In particular, $T=\left(T_{1}, T_{2}\right)$ is disk-cyclic.

Proof. Choose $z, w \in \mathcal{H}$ and let $W$ be a neighborhood for zero in $\mathcal{H}$. Without loss of generality, we suppose the diameter of $W$ is 1 , that is, $W=\{x \in \mathcal{H},\|x\|<1\}$. By the density of $\mathcal{X}$ and $\mathcal{Y}$ in $\mathcal{H}$, there are $x \in \mathcal{X}, y \in \mathcal{Y}$ such that

$$
\|z-x\|<\frac{1}{4} \text { and }\|w-y\|<\frac{1}{4}
$$

Denote $u=x+1 / \alpha S_{k} y \in \mathcal{H}$ for some $k \in \mathbb{N}$ and $0<\alpha \leq 1$, which are determined later. Using the assumptions in (1) and (2), there exists a positive integer $N$ such that

$$
\left\|T_{1}^{m_{k}} T_{2}^{n_{k}} S_{k} y-y\right\|<\frac{1}{4},\left\|S_{k} y\right\| \leq \frac{1}{4} \text { and }\left\|T_{1}^{m_{k}} T_{2}^{n_{k}} x\right\|\left\|S_{k} y\right\|<\frac{1}{8}
$$

for all $k>N$ and $x \in \mathcal{X}, y \in \mathcal{Y}$.

On the one hand, if $\left\|S_{k} y\right\| \neq 0$, fix $\alpha=4\left\|S_{k} y\right\| \leq 1$ and then

$$
\alpha\left\|T_{1}^{m_{k}} T_{2}^{n_{k}} x\right\|=4\left\|T_{1}^{m_{k}} T_{2}^{n_{k}} x\right\|\left\|S_{k} y\right\|<\frac{1}{2} .
$$


Based on the above inequalities, we obtain that

$$
\|z-u\|=\left\|z-x-1 / \alpha S_{k} y\right\| \leq\|z-x\|+1 / \alpha\left\|S_{k} y\right\|<1,
$$

and

$$
\begin{aligned}
& \left\|\alpha T_{1}^{m_{k}} T_{2}^{n_{k}} u-w\right\|=\left\|\alpha T_{1}^{m_{k}} T_{2}^{n_{k}}\left(x+1 / \alpha S_{k} y\right)-w\right\| \\
& \leq\left\|\alpha T_{1}^{m_{k}} T_{2}^{n_{k}} x\right\|+\left\|T_{1}^{m_{k}} T_{2}^{n_{k}} S_{k} y-y\right\|+\|y-w\| \\
& <\frac{1}{2}+\frac{1}{4}+\frac{1}{4}=1 .
\end{aligned}
$$

The above inequalities verify that $z-u \in W$ and $\alpha T_{1}^{m_{k}} T_{2}^{n_{k}} u-w \in W$ for $u \in \mathcal{H}$, $0<\alpha \leq 1$ and $m_{k}, n_{k} \in \mathbb{N}$ for $k>N$.

On the other hand, if $\left\|S_{k} y\right\|=0$ and since $T_{1}^{m_{k}} T_{2}^{n_{k}} S_{k} y \rightarrow y, k \rightarrow \infty$, then

$$
\begin{aligned}
\|y\| & \leq\left\|T_{1}^{m_{k}} T_{2}^{n_{k}} S_{k} y-y\right\|+\left\|T_{1}^{m_{k}} T_{2}^{n_{k}} S_{k} y\right\| \\
& \leq\left\|T_{1}^{m_{k}} T_{2}^{n_{k}} S_{k} y-y\right\|+\left\|T_{1}^{m_{k}} T_{2}^{n_{k}}\right\|\left\|S_{k} y\right\| \rightarrow 0, k \rightarrow \infty .
\end{aligned}
$$

That is, $y=0$. Hence $u=x+1 / \alpha S_{k} y=x$ and $\|w-y\|=\|w\|<1 / 4$. It's clear that $z-u=z-x \in W$. After that, we choose $0<\alpha_{0} \leq 1$ small enough, such that $\alpha_{0}\left\|T_{1}^{m_{k}} T_{2}^{n_{k}} x\right\|<1 / 4$. We deduce that

$$
\begin{aligned}
& \left\|\alpha_{0} T_{1}^{m_{k}} T_{2}^{n_{k}} u-w\right\|=\left\|\alpha_{0} T_{1}^{m_{k}} T_{2}^{n_{k}} x-w\right\| \\
& \leq\left\|\alpha_{0} T_{1}^{m_{k}} T_{2}^{n_{k}} x\right\|+\|w\|<1 / 4+1 / 4<1 .
\end{aligned}
$$

That means $\alpha_{0} T_{1}^{m_{k}} T_{2}^{n_{k}} u-w \in W$ for $0<\alpha_{0} \leq 1$.

In sum, under both cases, employing Theorem $2.3(1)$ the tuple $T=\left(T_{1}, T_{2}\right)$ is disk-cyclic.

Proposition 2.5. (Codisk-Cyclicity Criterion for tuples) Let $\mathcal{H}$ be a separable infinite dimensional Hilbert space and $T=\left(T_{1}, T_{2}\right)$ be a pair of commuting continuous linear mappings on $\mathcal{H}$. If there exist two dense subsets $\mathcal{X}, \mathcal{Y}$ in $\mathcal{H}$, a pair of strictly increasing positive integer sequences $\left(m_{k}\right)_{k \in \mathbb{N}}$ and $\left(n_{k}\right)_{k \in \mathbb{N}}$ and a sequence of mappings $S_{k}$ (not necessary bounded) such that $S_{k}(\mathcal{Y}) \subset \mathcal{Y}$ and

(1) $T_{1}^{m_{k}} T_{2}^{n_{k}} S_{k} y \rightarrow y, k \rightarrow \infty$ for all $y \in \mathcal{Y}$ and $\lim _{k \rightarrow \infty}\left\|T_{1}^{m_{k}} T_{2}^{n_{k}} x\right\|=0$ for all $x \in \mathcal{X}$.

(2) $\lim _{k \rightarrow \infty}\left\|T_{1}^{m_{k}} T_{2}^{n_{k}} x\right\|\left\|S_{k} y\right\|=0$ for all $x \in \mathcal{X}, y \in \mathcal{Y}$.

Then we say that $T=\left(T_{1}, T_{2}\right)$ satisfies the Codisk-Cyclicity Criterion. In particular, $T=\left(T_{1}, T_{2}\right)$ is codisk-cyclic.

Proof. Let $z, w \in \mathcal{H}$ and $W$ be a neighborhood for zero in $\mathcal{H}$. Without loss of generality, we also assume the diameter of $W$ is 1 , that is, $W=\{x \in \mathcal{H},\|x\| \leq 1\}$. By the density of $\mathcal{X}$ and $\mathcal{Y}$ in $\mathcal{H}$, there are $x \in \mathcal{X}, y \in \mathcal{Y}$ such that

$$
\|z-x\|<\frac{1}{4} \text { and }\|w-y\|<\frac{1}{4} \text {. }
$$

Denote $u=x+1 / \beta S_{k} y$ for some $k \in \mathbb{N}$ and $\beta \geq 1$, which are determined later. Using the assumptions in (1) and (2), there is a positive integer $N$ such that

$$
\left\|T_{1}^{m_{k}} T_{2}^{n_{k}} S_{k} y-y\right\|<\frac{1}{4},\left\|T_{1}^{m_{k}} T_{2}^{n_{k}} x\right\| \leq \frac{1}{4} \text { and }\left\|T_{1}^{m_{k}} T_{2}^{n_{k}} x\right\|\left\|S_{k} y\right\|<\frac{1}{8}
$$


for all $k>N$ and $x \in \mathcal{X}, y \in \mathcal{Y}$.

On the one hand, if $\left\|T_{1}^{m_{k}} T_{2}^{n_{k}} x\right\| \neq 0$, fix $\beta=\left(4\left\|T_{1}^{m_{k}} T_{2}^{n_{k}} x\right\|\right)^{-1} \geq 1$ and then

$$
\frac{1}{\beta}\left\|S_{k} y\right\|=4\left\|T_{1}^{m_{k}} T_{2}^{n_{k}} x\right\|\left\|S_{k} y\right\|<\frac{1}{2} .
$$

Based on the above inequalities, we obtain that

$$
\|z-u\|=\left\|z-x-1 / \beta S_{k} y\right\| \leq\|z-x\|+1 / \beta\left\|S_{k} y\right\|<1,
$$

and

$$
\begin{aligned}
& \left\|\beta T_{1}^{m_{k}} T_{2}^{n_{k}} u-w\right\|=\left\|\beta T_{1}^{m_{k}} T_{2}^{n_{k}}\left(x+1 / \beta S_{k} y\right)-w\right\| \\
& \leq\left\|\beta T_{1}^{m_{k}} T_{2}^{n_{k}} x\right\|+\left\|T_{1}^{m_{k}} T_{2}^{n_{k}} S_{k} y-y\right\|+\|y-w\| \\
& <\frac{1}{4}+\frac{1}{4}+\frac{1}{4}<1 .
\end{aligned}
$$

The above inequalities verify that $z-u \in W$ and $\beta T_{1}^{m_{k}} T_{2}^{n_{k}} u-w \in W$ for $\beta \geq 1$.

On the other hand, if $\left\|T_{1}^{m_{k}} T_{2}^{n_{k}} x\right\|=0$. Then choose $\beta>1$ large enough such that $1 / \beta\left\|S_{k} y\right\|<1 / 4$. Hence

$$
\|z-u\| \leq\|z-x\|+\frac{1}{\beta}\left\|S_{k} y\right\|<\frac{1}{4}+\frac{1}{4}<1 .
$$

That is, $z-u \in W$. Moreover,

$$
\begin{aligned}
\left\|\beta T_{1}^{m_{k}} T_{2}^{n_{k}} u-w\right\| & =\left\|\beta T_{1}^{m_{k}} T_{2}^{n_{k}}\left(x+1 / \beta S_{k} y\right)-w\right\| \\
& =\left\|T_{1}^{m_{k}} T_{2}^{n_{k}} S_{k} y-w\right\| \\
& \leq\left\|T_{1}^{m_{k}} T_{2}^{n_{k}} S_{k} y-y\right\|+\|y-w\| \\
& <\frac{1}{4}+\frac{1}{4}<1 .
\end{aligned}
$$

That means $\beta T_{1}^{m_{k}} T_{2}^{n_{k}} u-w \in W$. From Theorem 2.3 (2), we deduce the tuple $T=\left(T_{1}, T_{2}\right)$ is codisk-cyclic. This ends the proof.

For further use, we cite the definition for conjugacy from [7].

Definition 2.6. [7, Definition 1.5] Let $\widetilde{S}: Y \rightarrow Y$ and $\widetilde{T}: X \rightarrow X$ be two dynamical systems on Banach spaces $X$ and $Y$. Then $\widetilde{T}$ is called conjugate to $\widetilde{S}$ if there exists a homeomorphism $\phi: Y \rightarrow X$ such that $\widetilde{T} \circ \phi=\phi \circ \widetilde{S}$.

Concerning the disk-cyclicity and codisk-cyclicity, the following proposition holds.

Proposition 2.7. Disk-cyclicity (Codisk-cyclicity) for an operator $\widetilde{T} \in \mathcal{B}(\mathcal{H})$ is preserved under conjugacy. 


\section{Disk-cyclicity of the tuple $\left(C_{\omega_{1}, \varphi_{1}}^{*}, C_{\omega_{2}, \varphi_{2}}^{*}\right)$}

In this section, we mainly discover some sufficient conditions for the disk-cyclicity of the tuple $T=\left(C_{\omega_{1}, \varphi_{1}}^{*}, C_{\omega_{2}, \varphi_{2}}^{*}\right)$ on the Hilbert space $\mathcal{H}$. Firstly, we use the sets $A, B$ to state our main theorem.

Theorem 3.1. Let $\omega_{1}(z), \omega_{2}(z)$ be two nonzero complex-valued functions for all $z \in \mathbb{D}$ and $\varphi_{1}(z), \varphi_{2}(z)$ be two automorphisms in $\mathbb{D}$ satisfying (2.2). Suppose

$$
M=\sup _{z \in \mathbb{D}} \sup _{n \in \mathbb{Z}}\left\|k_{\left(\varphi_{1}\right)_{n} \circ\left(\varphi_{2}\right)_{n}(z)}\right\|<\infty .
$$

If the sets $A$ and $B$ have limit points in $\mathbb{D}$, then the tuple $T=\left(C_{\omega_{1}, \varphi_{1}}^{*}, C_{\omega_{2}, \varphi_{2}}^{*}\right)$ is diskcyclic on $\mathcal{H}$.

Proof. We will use Proposition 2.4 to prove the tuple $T=\left(C_{\omega_{1}, \varphi_{1}}^{*}, C_{\omega_{2}, \varphi_{2}}^{*}\right)$ is diskcyclic. Let $S_{A}=\operatorname{span}\left\{k_{z}: z \in A\right\}$ and $S_{B}=\operatorname{span}\left\{k_{z}: z \in B\right\}$. Then $\overline{S_{A}}=$ $\overline{S_{B}}=\mathcal{H}$, that is, the sets $S_{A}$ and $S_{B}$ are dense in $\mathcal{H}$. For the readers' convenience, we now get down to the details. If $f \in \mathcal{H}$ is orthogonal to $k_{z}$ for every $z \in S_{A}$, then $f(z)=\left\langle f, k_{z}\right\rangle$. Since the set $A$ has limit point in $\mathbb{D}$, hence the identity theorem for holomorphic functions implies that $f$ vanishes identically on $\mathcal{H}$. That is, $\left(S_{A}\right)^{\perp}=\{0\}$. Hence $\overline{S_{A}}=\mathcal{H}$. Similarly, $\overline{S_{B}}=\mathcal{H}$.

Let $\mathcal{X}=S_{A}$ and $\mathcal{Y}=S_{B}$, which are dense subsets of the Hilbert space $\mathcal{H}$. Since $\varphi_{1}$ and $\varphi_{2}$ are two automorphisms on $\mathbb{D}$, thus $\varphi_{1}^{-1}$ and $\varphi_{2}^{-1}$ exist on $\mathbb{D}$. Further, (2.2) implies that

$$
\varphi_{1}^{-1} \circ \varphi_{2}^{-1}=\varphi_{2}^{-1} \circ \varphi_{1}^{-1}, \omega_{1}=\omega_{1} \circ \varphi_{2}^{-1} \text { and } \omega_{2}=\omega_{2} \circ \varphi_{1}^{-1} .
$$

Note $T_{i}=C_{\omega_{i}, \varphi_{i}}^{*}$ for $i=1,2$. We observe from (2.3) that

$$
\begin{aligned}
T_{2}^{n} T_{1}^{n} k_{z}= & {\left[\Pi_{j=0}^{n-1}\left(\overline{\left(\omega_{1} \circ\left(\varphi_{1}\right)_{j}\right)(z)} \cdot \overline{\left(\omega_{2} \circ\left(\varphi_{2}\right)_{j}\right)(z)}\right)\right] } \\
& \cdot k_{\left(\varphi_{1}\right)_{n} \circ\left(\varphi_{2}\right)_{n}(z)}, n \geq 1 .
\end{aligned}
$$

To find the right inverse of $T_{2} T_{1}$, the proof falls into two cases according to the set $G_{B}=\left\{k_{z}: z \in B\right\}$ is linearly independent or not.

Case (i) Assume that $G_{B}$ is a linearly independent set. Define the operator $S: G_{B} \rightarrow \mathcal{H}$ by

$$
S k_{z}=\overline{\left[\left(\omega_{1} \circ \varphi_{1}^{-1}(z)\right) \cdot\left(\omega_{2} \circ \varphi_{2}^{-1}(z)\right)\right]^{-1}} k_{\varphi_{2}^{-1} \circ \varphi_{1}^{-1}(z)^{\prime}} z \in \mathbb{D} .
$$

Employing (3.2), we derive $S^{n}$ on $G_{B}$ for all $n \geq 1$,

$$
S^{n} k_{z}=\prod_{j=1}^{n} \overline{\left[\omega_{1} \circ\left(\varphi_{1}\right)_{-j}(z) \cdot \omega_{2} \circ\left(\varphi_{2}\right)_{-j}(z)\right]^{-1}} k_{\left(\varphi_{2}\right)_{-n} \circ\left(\varphi_{1}\right)_{-n}(z)} .
$$

Since $G_{B}$ is linearly independent, we extend $S$ by linearity on the set $\mathcal{Y}=S_{B}=$ $\operatorname{span}\left\{k_{z}: z \in B\right\}$. Hence $S^{n}$ is well-defined on $\mathcal{Y}$ and satisfies $S^{n}(\mathcal{Y}) \subset \mathcal{Y}$ for all $n \geq 1$. The assumption verifies that

$$
\lim _{n \rightarrow \infty}\left\|S^{n} y\right\|=0 \text { for all } y \in \mathcal{Y} .
$$


By (2.2), we arrive at $\varphi_{1} \circ \varphi_{2}=\varphi_{2} \circ \varphi_{1}, \omega_{2}=\omega_{2} \circ \varphi_{1}$ and it yields that

$$
\begin{aligned}
& T_{2} T_{1} S k_{z}=T_{2} T_{1}\left(\overline{\left[\left(\omega_{1} \circ \varphi_{1}^{-1}(z)\right) \cdot\left(\omega_{2} \circ \varphi_{2}^{-1}(z)\right)\right]^{-1}} k_{\varphi_{2}^{-1} \circ \varphi_{1}^{-1}(z)}\right) \\
& =T_{2}\left(\overline{\left[\omega_{2} \circ \varphi_{2}^{-1} \circ \varphi_{1}(z)\right]^{-1}} k_{\varphi_{2}^{-1}(z)}\right) \\
& =\overline{\omega_{2}(z)\left[\omega_{2} \circ \varphi_{2}^{-1} \circ\left(\varphi_{1} \circ \varphi_{2}\right)(z)\right]^{-1}} k_{z} \\
& =\overline{\omega_{2}(z)\left[\omega_{2} \circ \varphi_{2}^{-1} \circ\left(\varphi_{2} \circ \varphi_{1}\right)(z)\right]^{-1}} k_{z} \\
& =\overline{\omega_{2}(z)\left[\omega_{2}\left(\varphi_{1}(z)\right)\right]^{-1}} k_{z} \\
& =\overline{\omega_{2}(z)\left[\omega_{2}(z)\right]^{-1}} k_{z} \\
& =k_{z} \text {. }
\end{aligned}
$$

That is, $T_{2} T_{1} S=I d \mathcal{Y}$ and $T_{2}^{n} T_{1}^{n} S^{n}$ is the identity on $\mathcal{Y}$ for each $n \geq 1$. Hence

$$
T_{2}^{n} T_{1}^{n} S^{n} y \rightarrow y, n \rightarrow \infty, \text { for every } y \in \mathcal{Y}=S_{B}
$$

In addition, by the conditions and (3.1), we conclude that

$$
\begin{aligned}
& \lim _{n \rightarrow \infty}\left\|T_{2}^{n} T_{1}^{n} k_{y}\right\|\left\|S^{n} k_{z}\right\| \\
= & \lim _{n \rightarrow \infty}\left\|\left[\Pi_{j=0}^{n-1}\left(\overline{\left(\omega_{1} \circ\left(\varphi_{1}\right)_{j}\right)(y)} \cdot \overline{\left(\omega_{2} \circ\left(\varphi_{2}\right)_{j}\right)(y)}\right)\right] k_{\left(\varphi_{1}\right)_{n} \circ\left(\varphi_{2}\right)_{n}(y)}\right\| \\
\cdot & \left\|\Pi_{j=1}^{n} \overline{\left[\omega_{1} \circ\left(\varphi_{1}\right)_{-j}(z) \cdot \omega_{2} \circ\left(\varphi_{2}\right)_{-j}(z)\right]^{-1}} k_{\left(\varphi_{2}\right)_{-n} \circ\left(\varphi_{1}\right)_{-n}(z)}\right\| \\
\leq & M^{2} \sup _{n \in \mathbb{N}}\left|\Pi_{j=0}^{n-1}\left(\overline{\left(\omega_{1} \circ\left(\varphi_{1}\right)_{j}\right)(y)} \cdot \overline{\left(\omega_{2} \circ\left(\varphi_{2}\right)_{j}\right)(y)}\right)\right| \\
\cdot & \lim _{n \rightarrow \infty}\left|\Pi_{j=1}^{n} \overline{\left[\omega_{1} \circ\left(\varphi_{1}\right)_{-j}(z) \cdot \omega_{2} \circ\left(\varphi_{2}\right)_{-j}(z)\right]^{-1}}\right| \\
= & 0, \text { for } y \in A, z \in B .
\end{aligned}
$$

The above inequalities lead that

$$
\lim _{n \rightarrow \infty}\left\|T_{2}^{n} T_{1}^{n} x\right\|\left\|S^{n} y\right\|=0 \text {, for every } x \in \mathcal{X} \text { and } y \in \mathcal{Y}
$$

Depending on (3.5)-(3.7) and Proposition 2.4, the tuple $T=\left(C_{\omega_{1}, \varphi_{1}}^{*}, C_{\omega_{2}, \varphi_{2}}^{*}\right)$ fulfils the Disk-Cyclicity Criterion for tuples, hence $T=\left(C_{\omega_{1}, \varphi_{1}}^{*}, C_{\omega_{2}, \varphi_{2}}^{*}\right)$ is disk-cyclic on $\mathcal{H}$.

Case (ii). Assume that $G_{B}=\left\{k_{z}: z \in B\right\}$ is not necessarily linearly independent. In this case, we adapt the method used by Godefroy and Shapiro in [5, Theorem 4.5]. Consider a countable dense subset

$$
B_{1}=\left\{w_{n} \in \mathbb{D}: n \geq 1\right\}
$$

of $B$ and find a sequence $\left\{z_{n}\right\}$ by mathematical induction. Let $z_{1}=w_{1}$ and denote

$$
B_{2}=B_{1} \backslash\left\{w \in B_{1}: k_{w} \in \operatorname{span}\left\{k_{z_{1}}\right\}\right\} .
$$

Denote the first element of $B_{2}$ by $z_{2}$ and let

$$
B_{3}=B_{2} \backslash\left\{w \in B_{2}: k_{w} \in \operatorname{span}\left\{k_{z_{1}}, k_{z_{2}}\right\}\right\} .
$$


The infinite dimensionality of $\mathcal{H}$ insures the process never terminates and thus an infinite subset $L=\left\{z_{n} \in \mathbb{D}: n \geq 1\right\}$ of $B$ is obtained. The corresponding set of kernel functions $H_{L}=\left\{k_{z}: z \in L\right\}$ is linearly independent and is dense in $\mathcal{H}$. Then the operator $S$ can be defined exactly as above, just replacing $G_{B}$ by $H_{L}$. Consequently, the Disk-Cyclicity Criterion for tuples is also true in this case.

So, in both cases, the tuple $T=\left(C_{\omega_{1}, \varphi_{1}}^{*}, C_{\omega_{2}, \varphi_{2}}^{*}\right)$ is disk-cyclic. This finishes the proof.

Employing Theorem 3.1, we can obtain the disk-cyclicity of the tuple $\left(M_{\omega_{1}}^{*}, M_{\omega_{2}}^{*}\right)$ immediately.

Corollary 3.2. Let $\omega_{1}(z), \omega_{2}(z)$ be two nonzero complex-valued functions for all $z \in \mathbb{D}$. Denote the sets

$$
\begin{aligned}
& \widetilde{A}=\left\{z \in \mathbb{D}: \text { the sequence }\left\{\left(\omega_{1}(z) \omega_{2}(z)\right)^{n}\right\}_{n} \text { is bounded }\right\}, \\
& \widetilde{B}=\left\{z \in \mathbb{D}: \lim _{n \rightarrow \infty} \frac{1}{\left(\omega_{1}(z) \omega_{2}(z)\right)^{n}}=0\right\} .
\end{aligned}
$$

If the sets $\widetilde{A}$ and $\widetilde{B}$ have limit points in $\mathbb{D}$, then the tuple $\left(M_{\omega_{1}}^{*}, M_{\omega_{2}}^{*}\right)$ is disk-cyclic on $\mathcal{H}$.

Proof. Let $\varphi_{1}(z)=\varphi_{2}(z)=z$ in Theorem 3.1. It is evident that $\sup _{z \in \mathbb{D}}\left\|k_{z}\right\|<\infty$ emerged in (3.1) holds. Then the desired result follows from Theorem 3.1.

We show an example to account for Corollary 3.2.

Example 3.3. Let $w_{1}(z)=z$ and $w_{2}(z)=z+8$. Then

$$
\begin{aligned}
& \{x: 0 \leq x<\sqrt{17}-4\} \subseteq\left\{z \in \mathbb{D}: \text { the sequence }\left\{(z(z+8))^{n}\right\}_{n} \text { is bounded }\right\}, \\
& \{x:-1<x<-4+\sqrt{14}\} \subseteq\left\{z \in \mathbb{D}: \lim _{n \rightarrow \infty} \frac{1}{(z(z+8))^{n}}=0\right\} .
\end{aligned}
$$

The sets $\widetilde{A}$ and $\widetilde{B}$, apparently, have limit points in $\mathbb{D}$. The tuple $\left(M_{\omega_{1}}^{*}, M_{\omega_{2}}^{*}\right)$ is disk-cyclic due to Corollary 3.2.

For $a \in \mathbb{D}$, an automorphism $\phi_{a}(z)$ of $\mathbb{D}$ is defined by

$$
\phi_{a}(z)=\frac{a-z}{1-\bar{a} z}, \quad z \in \mathbb{D} .
$$

There are so many spaces that contain $\phi_{a}$, such as the Hardy space, Bergman space and Dirichlet space. These spaces are called automorphism invariant spaces. As we all know, all holomorphic self-maps of the unit disk $\mathbb{D}$ are dived into classes of elliptic and nonelliptic. The elliptic map is conjugate to a rotation $z \rightarrow \lambda z$ for some $\lambda \in \mathbb{C}$ with $|\lambda|=1$.

If $\varphi_{1}$ and $\varphi_{2}$ are two elliptic disk automorphisms satisfying $\varphi_{1} \circ \varphi_{2}=\varphi_{2} \circ \varphi_{1}$, then their interior fixed points are identical. Indeed, if $\varphi_{1}\left(z_{1}\right)=z_{1} \in \mathbb{D}$ and $\varphi_{2}\left(z_{2}\right)=z_{2} \in \mathbb{D}$, then

$$
\varphi_{1} \circ \varphi_{2}\left(z_{2}\right)=\varphi_{2} \circ \varphi_{1}\left(z_{2}\right) \Rightarrow \varphi_{1}\left(z_{2}\right)=\varphi_{2}\left(\varphi_{1}\left(z_{2}\right)\right) \Rightarrow \varphi_{1}\left(z_{2}\right)=z_{2} \Rightarrow z_{1}=z_{2} .
$$


Remark 3.4. For general case, if $\varphi_{1}$ and $\varphi_{2}$ have interior fixed points in $\mathbb{D}$ and satisfy $\varphi_{1} \circ \varphi_{2}=\varphi_{2} \circ \varphi_{1}$, then they have the same interior fixed points.

Theorem 3.5. Suppose that $\mathcal{H}$ is automorphism invariant. Let $\omega_{1}(z), \omega_{2}(z)$ be two nonzero complex-valued functions for all $z \in \mathbb{D}$ and $\varphi_{1}, \varphi_{2}$ be two elliptic disk automorphisms with an interior fixed point $a \in \mathbb{D}$ satisfying (2.2). If the sets $A$ and $B$ have limit points in $\mathbb{D}$, then the tuple $T=\left(C_{\omega_{1}, \varphi_{1}}^{*}, C_{\omega_{2}, \varphi_{2}}^{*}\right)$ is disk-cyclic on $\mathcal{H}$.

Proof. We divide the proof into two cases.

Case (i) Suppose the interior fixed point $a=0$. Then there are $\theta_{1}, \theta_{2} \in[0,2 \pi]$ such that

$$
\varphi_{1}(z)=e^{i \theta_{1}} z, \varphi_{2}(z)=e^{i \theta_{2}} z
$$

It yields that

$$
\left(\varphi_{2}\right)_{n} \circ\left(\varphi_{1}\right)_{n}(z)=e^{i n \theta_{1}} e^{i n \theta_{2}} z .
$$

Hence the iterate $\left\{\left(\varphi_{2}\right)_{n} \circ\left(\varphi_{1}\right)_{n}: n \in \mathbb{Z}\right\} \subseteq z \partial \mathbb{D}$. Since $z \partial \mathbb{D}$ is compact subset of $\mathbb{D}$, thus

$$
\left(f\left(\left(\varphi_{2}\right)_{n} \circ\left(\varphi_{1}\right)_{n}\right)\right)_{n \in \mathbb{Z}}
$$

is a bounded sequence for $f \in \mathcal{H} \cap H(\mathbb{D})$. By the uniform boundedness principle, we get that

$$
M=\sup _{z \in \mathbb{D}} \sup _{n \in \mathbb{Z}}\left\|k_{\left(\varphi_{2}\right)_{n} \circ\left(\varphi_{1}\right)_{n}}\right\|<\infty .
$$

Employing (3.9) and Theorem 3.1, the tuple $T=\left(C_{\omega_{1}, \varphi_{1}}^{*}, C_{\omega_{2}, \varphi_{2}}^{*}\right)$ satisfies the DiskCyclicity Criterion for tuples.

Case (ii) If $a \neq 0$ is the interior fixed point of $\varphi_{i}(i=1,2)$. We notice that $\mathcal{H}$ is automorphism invariant. Let

$$
\widetilde{\varphi_{1}}=\phi_{a} \circ \varphi_{1} \circ \phi_{a}^{-1}, \widetilde{\varphi_{2}}=\phi_{a} \circ \varphi_{2} \circ \phi_{a}^{-1}
$$

be two automorphisms with the interior fixed point zero, and let

$$
\widetilde{\omega_{1}}=\omega_{1} \circ \phi_{a}^{-1}, \widetilde{\omega_{2}}=\omega_{2} \circ \phi_{a}^{-1}
$$

be two multipliers of $\mathcal{H}$, where $\phi_{a}$ is the automorphism provided in (3.8). The disk-cyclicity of the tuple $\left(C_{\widetilde{\omega_{1}}, \widetilde{\varphi_{1}}}^{*}, C_{\omega_{2}, \widetilde{\varphi_{2}}}^{*}\right)$ on $\mathcal{H}$ follows from Case (i), where $C_{\widetilde{\omega}_{i}, \widetilde{\varphi}_{i}}=C_{\phi_{a}}^{-1} \circ C_{\omega_{1}, \varphi_{1}} \circ C_{\phi_{a}}$ for $i=1,2$. Finally, since $C_{\omega_{i}, \varphi_{i}}$ is conjugate $C_{\widetilde{\omega}_{i}, \widetilde{\varphi}_{i}}$ for $i=1,2$, and by Proposition 2.7, we obtain the desired result. This completes the proof.

Example 3.6. Take two elliptic disk automorphisms $\varphi_{1}(z)=i z, \varphi_{2}(z)=-i z$ with an interior fixed point $a=0 \in \mathbb{D}$ and $w_{1}(z)=z^{4}, w_{2}(z)=z^{4}+4$. The sets $A$ and $B$ are

$$
\begin{aligned}
& A=\left\{z \in \mathbb{D}: \text { the sequence }\left\{z^{4 n}\left(z^{4}+4\right)^{n}\right\}_{n} \text { is bounded }\right\}, \\
& B=\left\{z \in \mathbb{D}: \lim _{n \rightarrow \infty} \frac{1}{z^{4 n}\left(z^{4}+4\right)^{n}}=0\right\} .
\end{aligned}
$$

Since $\left[0, \frac{1}{2}\right) \subseteq A$ and $\left(\frac{1}{\sqrt[4]{2}}, 1\right) \subseteq B$, hence $\left(C_{\omega_{1}, \varphi_{1}}^{*}, C_{\omega_{2}, \varphi_{2}}^{*}\right)$ is disk-cyclic on $\mathcal{H}$ from Theorem 3.5. 


\section{Codisk-cyclicity of the tuple $\left(C_{\omega_{1}, \varphi_{1}}^{*}, C_{\omega_{2}, \varphi_{2}}^{*}\right)$}

In this section, we turn our attention to study the codisk-cyclic tuple $T=\left(C_{\omega_{1}, \varphi_{1}}^{*}, C_{\omega_{2}, \varphi_{2}}^{*}\right)$ on $\mathcal{H}$. Since the proofs of the codisk-cyclicity are exactly the same as those in Section 2, thus we omit the details. The main results are based on the sets $C$ and $D$ and Proposition 2.5.

Theorem 4.1. Let $\omega_{1}(z), \omega_{2}(z)$ be two nonzero complex-valued functions for all $z \in \mathbb{D}$ and $\varphi_{1}(z), \varphi_{2}(z)$ be two automorphisms in $\mathbb{D}$ satisfying (2.2). Suppose

$$
M=\sup _{z \in \mathbb{D}} \sup _{n \in \mathbb{Z}}\left\|k_{\left(\varphi_{1}\right)_{n} \circ\left(\varphi_{2}\right)_{n}(z)}\right\|<\infty .
$$

If the sets $C$ and $D$ have limit points in $\mathbb{D}$, then the tuple $\left(C_{\omega_{1}, \varphi_{1}}^{*}, C_{\omega_{2}, \varphi_{2}}^{*}\right)$ is codisk-cyclic on $\mathcal{H}$.

In view of Theorem 4.1, the following description for the codisk-cyclic tuple $\left(M_{\omega_{1}}^{*}, M_{\omega_{2}}^{*}\right)$ holds.

Corollary 4.2. Let $\omega_{1}(z), \omega_{2}(z)$ be two nonzero complex-valued functions for all $z \in \mathbb{D}$. Denote the sets

$$
\begin{aligned}
& \widetilde{C}=\left\{z \in \mathbb{D}: \lim _{n \rightarrow \infty}\left(\omega_{1}(z) \omega_{2}(z)\right)^{n}=0\right\}, \\
& \widetilde{D}=\left\{z \in \mathbb{D}: \text { the sequence }\left\{\frac{1}{\left(\omega_{1}(z) \omega_{2}(z)\right)^{n}}\right\}_{n} \text { is bounded }\right\} .
\end{aligned}
$$

If the sets $\widetilde{C}$ and $\widetilde{D}$ have limit points in $\mathbb{D}$, then the tuple $\left(M_{\omega_{1}}^{*}, M_{\omega_{2}}^{*}\right)$ is codisk-cyclic on $\mathcal{H}$.

Theorem 4.3. Suppose that $\mathcal{H}$ is automorphism invariant. Let $\omega_{1}(z), \omega_{2}(z)$ be two nonzero complex-valued functions for all $z \in \mathbb{D}$ and $\varphi_{1}, \varphi_{2}$ be two elliptic disk automorphisms with an interior fixed point $a \in \mathbb{D}$ satisfying (2.2). If the sets $C$ and $D$ have limit points in $\mathbb{D}$, then the tuple $\left(C_{\omega_{1}, \varphi_{1}}^{*}, C_{\omega_{2}, \varphi_{2}}^{*}\right)$ is codisk-cyclic on $\mathcal{H}$.

Theorem 4.4. Suppose that $\mathcal{H}$ is automorphism invariant. Let $\omega_{1}(z), \omega_{2}(z)$ be two nonzero complex-valued functions for all $z \in \mathbb{D}$ and $\varphi_{1}, \varphi_{2}$ be two elliptic automorphism with an interior fixed point $a \in \mathbb{D}$ satisfying (2.2). Further assume that $\omega_{1}, \omega_{2}$ : $\mathbb{D} \rightarrow \mathbb{C}$ satisfy the inequality $\left|\omega_{1}(a) \omega_{2}(a)\right|<1$ and there is $0<\delta<1$ satisfying $\left|\omega_{1}(z) \omega_{2}(z)\right| \geq 1$ for all $|z|>1-\delta$, then the tuple $\left(C_{\omega_{1}, \varphi_{1}}^{*}, C_{\omega_{2}, \varphi_{2}}^{*}\right)$ is codisk-cyclic on $\mathcal{H}$.

Proof. As the similar argument used in Theorem 3.5. Since $\mathcal{H}$ is automorphism invariant, we can only prove for the case $a=0$. Then

$$
\varphi_{1}(z)=e^{i \theta_{1}} z, \varphi_{2}(z)=e^{i \theta_{2} z}
$$

for some $\theta_{1}, \theta_{2} \in[0,2 \pi]$. Following the ideas in the proof of Theorem 3.5(Case (i)), (3.9) holds.

On the one hand, since $\left|\omega_{1}(0) \omega_{2}(0)\right|<1$, there is a constant $0<r<1$ and a positive number $\widetilde{\delta} \in(0,1)$ such that

$$
\left|\omega_{1}(z) \omega_{2}(z)\right|<r<1 \text {, whenever }|z|<\widetilde{\delta} .
$$


Since $\left|\varphi_{i}(z)\right|=|z|$ for $i=1,2$. Thus if $|z|<\widetilde{\delta}$, we have that

$$
\left|\Pi_{j=0}^{n-1} \omega_{1} \circ\left(\varphi_{1}\right)_{j}(z) \cdot \omega_{2} \circ\left(\varphi_{2}\right)_{j}(z)\right|<r^{n} \rightarrow 0, n \rightarrow \infty .
$$

Thus the set $\{z \in \mathbb{D}:|z|<\widetilde{\delta}\}$ is a subset of $C$ in Theorem 4.1.

On the other hand, since there is $0<\delta<1$ satisfying $\left|\omega_{1}(z) \omega_{2}(z)\right| \geq 1$ for all $|z|>1-\delta$, and $\left|\varphi_{i}^{-1}(z)\right|=|z|$ for $i=1,2$, hence if $|z|>1-\delta$, we conclude that

$$
\left|\Pi_{j=1}^{n} \omega_{1} \circ\left(\varphi_{1}\right)_{-j}(z) \cdot \omega_{2} \circ\left(\varphi_{2}\right)_{-j}(z)\right|^{-1} \leq 1, \text { for all } n \geq 1 \text {. }
$$

Therefore, the set $\{z \in \mathbb{D}:|z|>1-\delta\}$ is a subset of $D$ in Theorem 4.1. Since both $\{z \in \mathbb{D}:|z|<\widetilde{\delta}\}$ and $\{z \in \mathbb{D}:|z|>1-\delta\}$ have limit points in $\mathbb{D}$, then both $C$ and $D$ have limit points in $\mathbb{D}$. Employing (3.9) and Theorem 4.1, the tuple $\left(C_{\omega_{1}, \varphi_{1}}^{*}, C_{\omega_{2}, \varphi_{2}}^{*}\right)$ is codisk-cyclic. This completes the proof.

Remark 4.5. Example 3.6 indeed holds for Theorem 4.4. Since $\left|w_{1}(0) w_{2}(0)\right|=0<1$ and there is $0<\delta=1-\frac{1}{\sqrt[4]{3}}<1$ satisfying $\left|w_{1}(z) w_{2}(z)\right|=|z|^{4}\left|z^{4}+4\right| \geq$ $|z|^{4}(4-1)=3|z|^{4} \geq 1$ for all $|z|>1-\delta$. Hence the tuple $\left(C_{\omega_{1}, \varphi_{1}}^{*}, C_{\omega_{2}, \varphi_{2}}^{*}\right)$ is codiskcyclic on $\mathcal{H}$ from Theorem 4.4 .

\section{References}

[1] A.S. Atef, On G-cyclicity of operators, Thesis, The Islamic University of Gaza, 2007.

[2] F. Bayart, É. Matheron, Dynamics of Linear Operators, Cambridge University Press, 2009.

[3] T. Bermúdez, A. Bonilla, and A. Peris, On hypercyclicity and supercyclicity criteria, Bull. Austral. Math. Soc. 70 (1) (2004) 45-54.

[4] R.Y. Chen, Z.H. Zhou, Hypercyclicity of weighted composition operators on the unit ball of $\mathbb{C}^{N}$, J. Korean Math. Soc. 48 (2011) 969-984.

[5] G. Godefroy, J.H. Shapiro, Operators with dense, invariant, cyclic vector manifolds. J. Funct. Anal. 98 (1991) 229-269.

[6] K.G. Grosse-Erdmann, Hypercyclic and chaotic weighted shifts, Studia. Math. 139 (2000) 47-68.

[7] K.G. Grosse-Erdmann, A. Peris Manguillot, Linear Chaos, Springer, New York, 2011.

[8] Z.Z. Jamil, Cyclic phenomena of operators on Hilbert space, Ph.D Thesis, University of Baghdad, 2002.

[9] Z.Z. Jamil, M. Helal, Equivalent between the Criterion and the Three Open Set's Conditions in Disk-Cyclicity, Int. J. Contemp. Math. Sciences, 8 (2013) 257-261. 
[10] Z.Z. Jamil, A.G. Naoum, Disk-cyclic and weighted shifts operators, International J. of Math. Sci. and Engg. Appls. 7 (2013) 375-388.

[11] Z. Kamali, B.K. Robati, K.H. Shiraz, Cyclicity of the adjoint of weighted composition operators on the Hilbert space of analytic functions, Czech. Math. J. 61 (2011) 551-563.

[12] C. Kitai, Invariant Closed Sets for Linear Operators, Ph.D. Thesis, University of Toronto, 1982.

[13] Y.X. Liang and Z.H. Zhou, Hereditarily hypercyclicity and supercyclicity of weighted shifts, J. Korean Math. Soc. 51 (2) (2014) 363-382.

[14] Y.X. Liang and Z.H. Zhou, Disjoint supercyclic powers of weighted shifts on weighted sequence spaces, Turkish J. Math. 38 (2014) 1007-1022.

[15] Y.X. Liang and Z.H. Zhou, Hypercyclic behaviour of multiples of composition operators on the weighted Banach space, Bull. Belg. Math. Soc. Simon Stevin, 21(3) (2014) 385-401.

[16] Y.X. Liang and Z.H. Zhou,Supercyclic tuples of the adjoint weighted composition operators on Hilbert spaces, Bull. Iranian Math. Soc. 41(1)(2015) 121139.

[17] Y.X. Liang and Z.H. Zhou, Disk-cyclicity and codisk-cyclicity of certain shift operators, Oper. Matrices, 9(4) (2015) 831-846.

[18] S. Rolewicz, On orbits of operators, Studia Math. 32 (1969) 17-22.

[19] R. Soltani, B.K. Robati, K. Hedayatian, Hypercyclic tuples of the adjoint of the weighted composition operators, Turk J. Math. 36 (2012) 452-462.

[20] H.N. Salas, Supercyclicity and weighted shifts, Studia Math. 135 (1999) 55-74.

[21] B. Yousefi, Supercyclicity of multiple weighted composition operators, Int. Math. Forum, 6 (1) (2011) 15-18.

[22] B. Yousefi, J. Izadi, Weighted composition operators and Supercyclicity Criterion, Int. J. Math. and Math. Sci. 2011 (2011), ID 514370, 5 pages.

[23] B. Yousefi, H. Rezaei, Hypercyclicity property of weighted composition operators. Proc. Amer. Math. Soc. 135 (2007) 3263-3271.

School of Mathematical Sciences, Tianjin Normal University,

Tianjin 300387, P.R. China.

email:liangyx1986@126.com

Department of Mathematics and Center for Applied Mathematics,

Tianjin University,

Tianjin 300072, P.R. China.

email:zehuazhoumath@aliyun.com;zhzhou@tju.edu.cn 